Gómez Ruiz, P. y Gil López, A.J. (2018). El estilo de aprendizaje y su relación con la educación entre pares. Revista de Investigación Educativa, 36(1), 221-237.

DOI: http://dx.doi.org/10.6018/rie.36.1.233731

\title{
El estilo de aprendizaje y su relación con la educación entre pares
}

\section{Learning style and its relationship with peer tutoring}

\author{
Pilar Gómez Ruiz* y Alfonso J. Gil López \\ * Equipo de Orientación Educativo Psicopedagógico, La Rioja (España). \\ * Departamento de Economía y Empresa. Universidad de la Rioja (España)
}

\begin{abstract}
Resumen
Los objetivos de esta investigación son: diagnosticar el Estilo de Aprendizaje (EA) del alumnado de la Educación Secundaria Obligatoria (ESO), conocer la percepción de este alumnado sobre la figura del compañero tutor, y analizar la relación entre el EA del alumnado y la figura del compañero tutor. Se plantean como hipótesis que no existen diferencias entre los Estilos de Aprendizaje del alumnado y las características del compañero tutor. El estudio empírico se llevó a cabo con estudiantes de todos los cursos de la ESO en 6 Centros en España. La recogida de datos se realizó con dos instrumentos: el CHAEA y un cuestionario diseñado por el propio equipo de investigación sobre compañero tutor. En los resultados se comprueba que el EA del alumnado es predominantemente reflexivo, que los alumnos consideran la figura del compañero tutor adecuada para su aprendizaje, y las mayores diferencias significativas se encuentran entre el Estilo activo y el compañero tutor.

Palabras clave: estilo de aprendizaje; compañero tutor; atención a la diversidad; enseñanza secundaria.
\end{abstract}

Correspondencia: Alfonso J. Gil López, alfonso.gil@unirioja.es Departamento de Economía y Empresa, Universidad de La Rioja, Edificio Quintiliano, C/La Cigüeña, 60, 26006, Logroño, La Rioja. 


\begin{abstract}
The objectives of this work are: to diagnose the Learning Style (LS) of the students of compulsory secondary education (ESO), to know their perception of the peer tutoring and to analyze the relationship between the students' LS and peer tutoring. The empirical study was conducted with students from all courses of the ESO in 6 school centers in Spain. Data collection was performed using two instruments: the CHAEA and a questionnaire designed by the team of research on peer tutor. In the results it is found that the students' $L S$ is predominantly reflective, students consider the figure of the peer tutor right for learning, and the largest significant differences were found between the active Style and peer tutor.

Keywords: learning style; peer tutoring; attention to diversity; secondary education.
\end{abstract}

\title{
Introducción
}

En la actualidad un gran reto docente es responder a la diversidad de estilos de aprender y las diferentes necesidades educativas de los alumnos en las aulas (Álvarez et al., 2002). Bajo esta perspectiva se plantea este trabajo, en el que analizados dos temas relacionados con el desarrollo del aprendizaje del alumnado que cursa Educación Secundaria Obligatoria (ESO) en España. Por un lado, su Estilo de Aprendizaje (EA) y, por otro, la educación entre pares o tutoría entre iguales.

El EA se ha definido como el conjunto de características psicológicas, rasgos cognitivos, afectivos y fisiológicos que suelen expresarse conjuntamente cuando una persona debe enfrentar una situación de aprendizaje (Alonso, Gallego y Honey, 1994). El EA se refiere a la forma que cada persona afronta la tarea de aprender (Moya, Hernández, Hernández y Cózar, 2011). El concepto de EA está relacionado, entonces, con la idea de que aprendizaje es un proceso activo, en el que propio discente elabora su aprendizaje (Armstrong, Cools y Sadler-Smith, 2012; Li y Armstrong, 2015). Por ello, el diagnóstico y análisis de los Estilos de Aprendizaje resultan especialmente importantes para alcanzar un aprendizaje efectivo. Pues, por un lado, se mejora la acción didáctica al ajustar el estilo de enseñar al estilo de aprender y, por otro, se desarrolla el EA al conocer las posibles deficiencias en el aprendizaje del alumno (Soflano, Connolli y Haney, 2015).

La literatura ha señalado diversos tipos de instrumentos para diagnosticar el EA de los estudiantes, en especial para los nativos en inglés, de entre los que se pueden destacar: el "Learning Style Inventory" (Dunn, Dunn y Price, 1975, 1989); el "Student Learning Styles Scales" (Riechmann y Grasha, 1974); el "Learning Style Delineantor" (Gregorc, 1982); y el “Learning Styles Inventory” (Kolb, 1976, 1984). De los instrumentos más conocidos en lengua española se encuentra el "Cuestionario Honey Alonso de Estilos de Aprendizaje" CHAEA (Alonso, 1992).

Siguiendo los estudios de Honey y Mumford (1986), Alonso (1992) llevó a cabo una investigación con los estudiantes universitarios de distintas especialidades académicas. De esta investigación, surgió el Cuestionario Honey Alonso de Estilos de Aprendizaje CHAEA que tiene un largo recorrido en los últimos años, son muy numerosos los estudios que utilizan el CHAEA como instrumento de análisis del Estilo de Aprendizaje del alumnado o del aprendiz (ver tabla 1). 
Tabla 1

Estudios sobre Estilos de Aprendizaje y CHAEA

\begin{tabular}{lcccc}
\hline Estudios & Activo & $\begin{array}{c}\text { Reflexivo } \\
\text { Media }\end{array}$ & Teórico & Pragmático \\
\hline Universitarios (Alonso, 1992) & 10.7 & 15.3 & 11.3 & 13.1 \\
Bachilleres (Adán, 2002) & 12.3 & 14.8 & 12.3 & 12.9 \\
Bachilleres (Santibáñez, Adán, Gil y Sáenz de & 12.9 & 14.3 & 12.7 & 12.9 \\
Jubera, 2004) & 12.1 & 14.2 & 12.2 & 12.7 \\
Matemáticas (Nevot, 2004) & 12.8 & 13.6 & 12.9 & 13.4 \\
Secundaria (Quintanal y Gallego, 2011) & 14.4 & 13.2 & 11.7 & 13.2 \\
PCPI (Gil y Sánchez, 2012) & 13.1 & 12.3 & 11.4 & 13.6 \\
PCPI y 4º curso de ESO (Antelm y Gil, 2013) & &
\end{tabular}

Nota: adaptado a partir de Gil y Sánchez (2012). Nota. CHAEA: Cuestionario Honey Alonso de Estilos de Aprendizaje. PCPI: Programa de Cualificación Profesional Inicial. ESO: Educación Secundaria Obligatoria.

Como se observa en la tabla 1 existe un predominio casi absoluto en los estudiantes de distintas especialidades en el EA reflexivo, en particular las medias más elevadas se observan en la investigación de Alonso (1992) con estudiantes Universitarios (15.3). Los estudiantes con un Estilo predominantemente activo son los que cursan algún Programa de Cualificación Profesional Inicial (PCPI) (Gil y Sánchez, 2012). Y los estudiantes con un Estilo predominantemente pragmático cursan algún PCPI y $4^{\circ}$ curso de ESO.

En un sentido amplio, la tutoría entre pares o iguales se entiende como parejas de personas, que pueden ser alumnos, familiares o profesores, que aprenden a partir de una interacción estructurada (Durán, 2006). Se trata, por tanto, de un método de aprendizaje cooperativo, un recurso didáctico que convierte el trabajo en grupo en aprendizaje (Durán y Monereo, 2012). El aprendizaje cooperativo se basa en la teoría del aprendizaje social, según esta teoría los discentes tienen más probabilidades de poseer alta autoeficacia para completar una tarea cuando saben que tendrán la asistencia de sus pares (Ormrod, 1999). Cuando se trabaja en cooperación con los demás, las personas adquieren estrategias de aprendizaje más eficaces y resuelven problemas con un mayor éxito (De Backer, Van Keer y Valcke, 2015; Gillies y Ashman, 2003). Con todo ello, el aprendizaje entre iguales se convierte en un eficaz instrumento de aprendizaje activo, en el que se considera la diversidad del alumnado para intervenir en los procesos de aprendizaje en común. Pero, como indica Topping (2000), parece necesario aplicar esta estrategia de aprendizaje de forma adecuada en las aulas. Para ello, la planificación de este tipo estrategias puede ayudar a evitar la transferencia de información errónea, que puede dificultar el aprendizaje del alumno tutorizado (Durán y Vidal, 2004).

Como se observa ambos temas, el EA y la tutoría entre pares, están relacionados. A través del desarrollo del EA y de la tutoría entre iguales, se facilita la atención a la diversidad y la mejora de los procesos de aprendizaje del alumnado. Como señalan Loke y Chow (2007), los estudiantes que participan en los procesos de tutoría entre 
iguales mejoran sus habilidades de aprendizaje y su capacidad reflexiva de pensamiento crítico, lo que implica una mejora en su EA. De la misma forma, un fortalecimiento de los Estilos de Aprendizaje de los estudiantes puede mejorar las experiencias con otros de sus pares (Rodríguez y Vázquez, 2013) y, con ello, avanzar en la educación entre iguales.

El estudio empírico se lleva a cabo con estudiantes de ESO. Esta etapa es crucial en el alumnado pues supone el comienzo de "la pubertad" (Fierro, 1993). Se trata de una etapa de transición entre la infancia y la edad adulta. Es una época en la que las personas tratan de crear su identidad y un sentido de autonomía. A nivel cognitivo se caracteriza por el pensamiento formal, que es un pensamiento hipotético, lógico y abstracto, de manera que el adolescente o adulto es capaz de pensar sobre las abstracciones y conceptos hipotéticos así como de especular mentalmente sobre lo real y lo posible. En esta etapa del ciclo vital las relaciones sociales están condicionadas por los tres contextos interrelacionados en los años escolares: la familia, la escuela y los iguales. Por estas razones, en esta etapa educativa es especialmente significativo el EA y la figura del compañero tutor como instrumentos de mejora de los procesos de aprendizaje.

\section{Método}

\section{Objetivos e hipótesis}

En esta investigación empírica se plantean cuatro objetivos: (1) analizar las propiedades psicométricas de las puntuaciones del instrumento de medida de las características del compañero tutor; (2) analizar el EA predominante del alumnado de la ESO; (3) analizar las respuestas de este alumnado en relación a la educación entre iguales; y (4) contrastar la relación entre el EA de este alumnado y sus respuestas relativas al compañero tutor. La tabla 2 recoge las hipótesis propuestas.

Tabla 2

Hipótesis propuestas sobre las variables relacionadas con la figura del compañero tutor y sobre las características de los Estilos de Aprendizaje

\begin{tabular}{ll}
\hline Hipótesis & \multicolumn{1}{c}{ Descripción de la hipótesis } \\
\hline H1 & $\begin{array}{l}\text { El instrumento de medida de la figura del compañero tutor presentará adecuadas } \\
\text { propiedades en cuanto a la fiabilidad de las puntuaciones y evidencias de validez de } \\
\text { estructura interna. }\end{array}$ \\
& $\begin{array}{l}\text { No Existen diferencias estadísticamente significativas entre las características del EA } \\
\text { del alumnado de ESO y las variables relacionadas con el compañero tutor denomi- }\end{array}$ \\
& nadas como "tareas de tutor". \\
H3 & $\begin{array}{l}\text { No Existen diferencias estadísticamente significativas entre las características del EA } \\
\text { del alumnado de ESO y las variables relacionadas con el compañero tutor denomi- } \\
\text { nadas como "beneficios como tutorizado". }\end{array}$
\end{tabular}


H4 No Existen diferencias estadísticamente significativas entre las características del EA del alumnado de ESO y las variables relacionadas con el compañero tutor denominadas como "beneficios como tutor".

H5 No Existen diferencias estadísticamente significativas entre las características del EA del alumnado de ESO y las variables relacionadas con el compañero tutor denominadas como "problemas como tutor".

Nota: EA: Estilo de Aprendizaje; ESO: Educación Secundaria Obligatoria.

\section{Participantes}

El estudio se realizó en una muestra de 6 Institutos de Enseñanza Secundaria Obligatoria de las Comunidades Autónomas de Aragón, Navarra y Valencia. La encuesta se llevó a cabo durante los meses de febrero y marzo de 2015, coincidiendo con el curso avanzado, de tal forma que el alumnado tuviera una experiencia que podría plasmar en las respuestas del cuestionario.

La muestra está formada por 302 estudiantes de la ESO, que se distribuyen por sexo, edad y nivel académico según señala la tabla 3.

Tabla 3

Características de la muestra de alumnado

\begin{tabular}{lll}
\hline Variables & $\mathbf{N}$ & $\mathbf{\%}$ \\
\hline Sexo & & \\
Hombre & 156 & 51.7 \\
Mujer & 146 & 48.3 \\
Edad & & \\
12 años & 29 & 9.6 \\
13 años & 61 & 20.2 \\
14 años & 80 & 26.5 \\
15 años & 74 & 24.5 \\
16 años & 37 & 12.2 \\
17 años & 14 & 5.6 \\
+17 años & 4 & 1.4 \\
Nivel académico (ESO) & & \\
$1^{\text {o }}$ & 37 & 12.3 \\
$2^{\underline{0}}$ & 81 & 26.8 \\
$3^{\underline{o}}$ & 94 & 31.1 \\
$4^{\underline{0}}$ & 90 & 29.8 \\
(N) & $(302)$ & $(100)$ \\
\hline
\end{tabular}

Nota: ESO: Educación Secundaria Obligatoria. 


\section{Variables e instrumento}

Las variables dependientes se corresponden con los Estilos de Aprendizaje (activo, reflexivo, teórico y pragmático) de los alumnos de la ESO y las variables independientes son las características que definen al compañero tutor recogidas en el cuestionario.

Para la recogida de datos se utilizaron dos instrumentos: el CHAEA y un cuestionario ad hoc que recoge las características del compañero tutor. El CHAEA (Alonso et al., 1994) toma como base los trabajos de Honey y Mumford (1986) y señala 4 estilos de aprendizaje: activo, reflexivo, teórico y pragmático. En la tabla 4 se recogen las características de cada uno de los estilos de aprendizaje.

Tabla 4

Relación entre las etapas del ciclo de aprendizaje y los Estilos de Aprendizaje según Honey y Mumford

\begin{tabular}{|c|c|c|}
\hline $\begin{array}{l}\text { Ciclo de } \\
\text { Aprendizaje }\end{array}$ & $\begin{array}{l}\text { Estilo de } \\
\text { Aprendizaje }\end{array}$ & Características del Estilo de Aprendizaje \\
\hline
\end{tabular}

Primer Nivel:

Experimentar $\quad$ Estilo Activo

Las personas que tienen preponderancia en Estilo activo se implican plenamente y sin prejuicios en nuevas experiencias. Son de mente abierta, nada escépticos y acometen con entusiasmo las tareas nuevas.

Segundo Nivel:

Reflexionar

Estilo Reflexivo

A los reflexivos les gusta considerar las experiencias y observarlas desde diferentes perspectivas. Recogen datos, analizándolos con detenimiento antes de llegar a alguna conclusión.

Los teóricos adaptan e integran las observaciones dentro

Tercer Nivel:

Concluir

Estilo Teórico

de sus teorías lógicas y complejas. Tienden a ser perfeccionistas. Integran los hechos en teorías coherentes.

El punto fuerte de las personas con predominancia en es-

Cuarto Nivel: $\quad$ Estilo

Planificar Pragmático tilo pragmático es la aplicación práctica de las ideas. Les gusta actuar rápidamente y con seguridad con aquellas ideas y proyectos que les atraen.

Nota: Fuente a partir Fleming, Mckee y Huntley-Moore (2011)

El cuestionario ad hoc fue diseñado por el propio equipo de investigación. Para la realización de este instrumento se revisaron numerosas investigaciones relativas al aprendizaje entre iguales (Cheng y Ku, 2009; De Backer, Van Keer y Valcke, 2012; De Backer et al., 2015; Ginsburg-Block y Fantuzzo, 1997; Rittschof y Griffin, 2001), lo que permitió conocer las principales características de esta figura, que se trasladaron al cuestionario. En el apartado de resultados se cometan su estructura factorial y se examina su fiabilidad y su validez. 


\section{Análisis de datos}

Se realizaron diferentes análisis estadísticos para llevar a cabo los objetivos del estudio. En primer lugar, se calculó la estructura interna del cuestionario mediante análisis de componentes principales con posterior rotación Varimax. La fiabilidad de las puntuaciones se estimó mediante el coeficiente alpha de Cronbach. También se calcularon coeficientes de correlación lineal de Pearson y estadísticos univariados. Finalmente se llevaron a cabo diferentes ANOVAS univariados. Para el análisis estadístico se utilizó el programa SPSS versión 22.

\section{Resultados}

\section{Propiedades psicométricas del instrumento de medida: evidencias de estructura interna y estimación de la fiabilidad}

En primer lugar se lleva a cabo un análisis de componentes principales con rotación Varimax. Los diferentes criterios utilizados para la determinación de la estructura dimensional de las puntuaciones que la mejor solución factorial se ajusta a factores que coinciden con los 4 grupos de facetas que se han denominado como: (1) tareas como tutor; (2) beneficios como tutorizado; (3) beneficios como tutor, y (4) problemas de tutorización (ver tabla 5).

Tabla 5

Análisis factorial de las características del compañero tutor

\begin{tabular}{|c|c|c|c|c|}
\hline Variables & $\begin{array}{c}\text { Tareas de } \\
\text { tutor }\end{array}$ & $\begin{array}{l}\text { Beneficios } \\
\text { tutorizado }\end{array}$ & $\begin{array}{c}\text { Beneficios } \\
\text { tutor }\end{array}$ & $\begin{array}{l}\text { Problemas } \\
\text { de tutoría }\end{array}$ \\
\hline Tareas de Ayuda & .843 & & & \\
\hline Tareas de Apoyo & .783 & & & \\
\hline Beneficios en comprensión & & .785 & & \\
\hline Beneficios en estudio & & .736 & & \\
\hline Beneficios en tareas & & .673 & & \\
\hline Beneficios en apoyo & & .582 & & \\
\hline Beneficios en tareas & & & .809 & \\
\hline Beneficios en comprensión & & & .605 & \\
\hline Beneficios en motivación & & & .593 & \\
\hline Problemas en comprensión & & & & .841 \\
\hline Problemas en ayuda & & & & .781 \\
\hline$\%$ Varianza & 20.891 & 18.645 & 13.988 & 13.378 \\
\hline$\%$ Varianza acumulada & 20.891 & 39.536 & 53.524 & 66.903 \\
\hline
\end{tabular}


Como se observa en la tabla 5, en el análisis de la varianza total explicada llevada a cabo se comprueba que las 4 primeras componentes resumen el 66.903 de la variabilidad total. Además, la medida Káiser-Meyer-Olkin resultó 0.763; mientras que el test de esfericidad de Bartlett resultó 835.214 ( $\mathrm{p}<.001)$, lo que señala que no es significativa la hipótesis nula de variables iniciales incorrelacionadas, por ello se puede llevar a cabo la aplicación del análisis factorial (Pérez, 2004).

Para estimar la fiabilidad de las puntuaciones en el cuestionario se utilizó el coeficiente alpha de Cronbach para las escalas de más de 2 ítems y la correlación de Pearson para las escalas con únicamente 2 ítems. Como se observa en la tabla 6, las alpha de Cronbach (Cronbach, 1951) son superiores a .700 como se exige en las etapas tempranas de la investigación (Nunnally, 1978). Y el coeficiente de Pearson es superior a .300 (tabla 7).

Tabla 6

Coeficiente alpha de Cronbach

\begin{tabular}{lcc}
\hline & Número de ítems & $\alpha$ de Cronbach \\
\hline Beneficios como tutorizado & 4 & .730 \\
Beneficios como tutor & 3 & .700 \\
\hline
\end{tabular}

Tabla 7

Correlación de Pearson

\begin{tabular}{lccc}
\hline & $\begin{array}{c}\text { Número de } \\
\text { ítems }\end{array}$ & $\begin{array}{c}\text { Correlación } \\
\text { de Pearson }\end{array}$ & $\mathrm{p}$ \\
\hline Tareas de tutor & 2 & .596 & $<.001$ \\
Problemas con la tutorización & 2 & .347 & $<.001$ \\
\hline
\end{tabular}

La validez de constructo se refiere a la exactitud con el que un instrumento de medida evalúa un atributo (García, 2003), con el constructo o variable no observable latente que se pretende medir. Para obtener evidencias de validez se utilizó las correlaciones de Pearson. Para cada dimensión se obtuvo la correlación entre cada uno de los ítems enunciados para su medida, tal como se observa en la tabla 8.

Como se observa en la tabla 8, las correlaciones medias son, en general, elevadas. Asimismo son muy elevadas las correlaciones de valor máximo. En cuanto a las correlaciones de valor mínimo no se encuentran valores pequeños. Con todo lo señalado se confirma la Hipótesis 1, el instrumento de medida presenta adecuadas propiedades en cuanto a la fiabilidad de las puntuaciones y evidencias de validez de estructura interna. 
Tabla 8

Correlaciones de Pearson

\begin{tabular}{lccc}
\hline & $\begin{array}{c}\text { Valor } \\
\text { mínimo }\end{array}$ & $\begin{array}{c}\text { Valor } \\
\text { medio }\end{array}$ & $\begin{array}{c}\text { Valor } \\
\text { máximo }\end{array}$ \\
\hline Beneficios como tutorizado & .280 & .404 & .452 \\
Beneficios como tutor & .257 & .336 & .428 \\
Tareas de tutor & .569 & .569 & .569 \\
Problemas con la tutorización & .347 & .347 & .347 \\
\hline
\end{tabular}

Nota: Todas las correlaciones resultaron estadísticamente significativas $\mathrm{p}<.01$.

\section{Estadísticos descriptivos del EA predominante del alumnado de la ESO}

En la tabla 9 se recogen los primeros resultados relativos a los estadísticos descriptivos del EA.

Tabla 9

Estilos de Aprendizaje del alumnado de la ESO

\begin{tabular}{lllll}
\hline & Activo & Reflexivo & Teórico & Pragmático \\
\hline $\mathrm{N}$ & 302 & 302 & 302 & 302 \\
Media & 12.33 & 13.34 & 12.47 & 12.83 \\
Mediana & 13 & 14 & 13 & 13 \\
Desviación típica & 3.31 & 3.01 & 3.05 & 2.76 \\
Mínimo & 2 & 2 & 1 & 5 \\
Máxima & 20 & 21 & 19 & 20 \\
\hline
\end{tabular}

Nota: ESO: Educación Secundaria Obligatoria.

En la tabla 9 se observa que el EA predominante del alumnado de la ESO es reflexivo, seguido del pragmático, del teórico y del activo. Las desviaciones típicas son similares en todos los casos.

\section{Estadísticos descriptivos de las respuestas del alumnado relacionadas con el apren- dizaje entre iguales}

En la tabla 10 se recogen resultados relativos a los estadísticos descriptivos que se relacionan con el estudio del compañero tutor. 
Tabla 10

Análisis descriptivo sobre las respuestas de los alumnos de la ESO en relación a su percepción sobre el compañero tutor

\begin{tabular}{|c|c|c|}
\hline Grupos de preguntas & Media & $\begin{array}{l}\text { Desviación } \\
\text { típica }\end{array}$ \\
\hline \multicolumn{3}{|l|}{ Tareas de Tutor } \\
\hline $\begin{array}{l}\text { Con regularidad ayudo a mis compañeros o compañeras a hacer sus } \\
\text { tareas o trabajos de clase. }\end{array}$ & 3.76 & 1.61 \\
\hline Con regularidad ayudo a mis compañeros o compañeras a estudiar & 3.35 & 1.61 \\
\hline \multicolumn{3}{|l|}{ Beneficios como tutorizado } \\
\hline $\begin{array}{l}\text { Cuando mis compañeros o compañeras me ayudan a estudiar com- } \\
\text { prendo mejor lo que estoy estudiando }\end{array}$ & 4.84 & 1.43 \\
\hline $\begin{array}{l}\text { Cuando estoy acompañado con otro compañero o compañera estudio } \\
\text { mucho mejor }\end{array}$ & 3.66 & 1.81 \\
\hline $\begin{array}{l}\text { Cuando mis compañeros o compañeras me ayudan a hacer los traba- } \\
\text { jos de clase los hago mucho mejor }\end{array}$ & 4.37 & 1.67 \\
\hline $\begin{array}{l}\text { Con regularidad me suelo ayudar de un compañero o compañera } \\
\text { para estudiar }\end{array}$ & 2.82 & 1.68 \\
\hline \multicolumn{3}{|l|}{ Beneficios como tutor } \\
\hline $\begin{array}{l}\text { Cuando ayudo a un compañero o compañera a hacer sus deberes } \\
\text { aprendo mejor }\end{array}$ & 4.77 & 1.56 \\
\hline $\begin{array}{l}\text { Cuando leo una actividad junto con un compañero o compañera en- } \\
\text { tiendo mejor la actividad }\end{array}$ & 4.52 & 1.59 \\
\hline $\begin{array}{l}\text { Me motiva mucho ayudar en el estudio o en las tareas de clase a un } \\
\text { compañero o compañera }\end{array}$ & 4.37 & 1.73 \\
\hline \multicolumn{3}{|l|}{ Problemas con la tutorización } \\
\hline $\begin{array}{l}\text { Cuando leo una actividad con un compañero o compañera entiendo } \\
\text { peor la actividad que estoy leyendo. }\end{array}$ & 3.39 & 1.90 \\
\hline $\begin{array}{l}\text { Cuando ayudo a un compañero o compañera a estudiar comprendo } \\
\text { peor lo que estoy estudiando. }\end{array}$ & 3.04 & 2.70 \\
\hline
\end{tabular}

Nota: ESO: Educación Secundaria Obligatoria.

Como se observa en los datos, la puntuación más alta (4.84) corresponde con la pregunta: "Cuando mis compañeros a compañeras me ayudan a estudiar comprendo mejor lo que estoy estudiando". También es relativamente elevada (4.77) la pregunta: "Cuando ayudo a un compañero o compañera a hacer sus deberes aprendo mejor". Asimismo, es relativamente elevada (4.52) la pregunta: "Cuando leo una actividad junto con un compañero o compañera entiendo mejor la actividad". La cuestión con menos puntuación (2.82) es: "Con regularidad me suelo ayudar de un compañero o compañera para estudiar", también tiene una baja puntuación las preguntas que hacen 
referencia a los problemas con la tutorización (3.39 y 3.04 respectivamente): “Cuando leo una actividad con un compañero o compañera entiendo peor la actividad que estoy leyendo" y "Cuando ayudo a un compañero o compañera a estudiar comprendo peor lo que estoy estudiando".

\section{Contraste de hipótesis de las relaciones entre el EA y las características de com- pañero tutor}

En la tabla 11 se recoge el análisis de varianza de las variables dependientes "Estilos de Aprendizaje" y las variables independientes "tareas de tutor".

Tabla 11

Análisis de varianza para las variables dependientes EA activo, reflexivo, teórico y pragmático, y las variables independientes relacionadas con las "tareas de tutor" del alumnado de la ESO

\begin{tabular}{lcccccc}
\hline EA & \multicolumn{3}{c}{ Tutor en tareas } & \multicolumn{3}{c}{ Tutor en estudio } \\
\cline { 2 - 7 } & GL & F & p & GL & F & p \\
\hline Activo & 6 & 1.134 & .343 & 6 & .123 & .994 \\
Reflexivo & 6 & .910 & .488 & 6 & 1.572 & .155 \\
Teórico & 6 & 1.016 & .415 & 6 & 1.383 & .221 \\
Pragmático & 6 & 1.152 & .332 & 6 & 1.055 & .390 \\
\hline
\end{tabular}

Nota: GL: Grados de Libertad; EA: Estilo de Aprendizaje. ESO: Educación Secundaria Obligatoria.

Como se observa en la tabla 11 no existen diferencias significativas entre las variables dependientes "Estilos de Aprendizaje" y las variables relacionadas con el compañero tutor, que hemos denominado como "tareas de tutor", por lo que aceptamos la Hipótesis 2.

En la tabla 12 recogemos el análisis de varianza de las variables dependientes "Estilos de Aprendizaje" y las variables independientes "beneficios como tutorizado".

Tabla 12

Análisis de varianza para la variable dependiente EA de aprendizaje activo, reflexivo, teórico y pragmático, y las variables independientes relacionadas con los "beneficios como tutorizado" del alumnado de la ESO

\begin{tabular}{lcccccccccccc}
\hline EA & \multicolumn{3}{c}{$\begin{array}{c}\text { Tutorización en } \\
\text { estudio }\end{array}$} & \multicolumn{3}{c}{$\begin{array}{c}\text { Tutorización en } \\
\text { acompanamiento }\end{array}$} & \multicolumn{2}{c}{$\begin{array}{c}\text { Tutorización en } \\
\text { trabajos }\end{array}$} & \multicolumn{3}{c}{$\begin{array}{c}\text { Tutorización en } \\
\text { ayuda }\end{array}$} \\
\cline { 2 - 14 } & GL & F & p & GL & F & p & GL & F & p & GL & F & p \\
\hline Activo & 6 & 2.965 & .008 & 6 & 2.940 & .008 & 6 & 2.940 & .008 & 6 & 1.166 & .325 \\
Reflexivo & 6 & 1.349 & .235 & 6 & 1.101 & .362 & 6 & 1.101 & .362 & 6 & 1.220 & .296 \\
Teórico & 6 & 3.342 & .001 & 6 & 2.632 & .017 & 6 & 2.632 & .017 & 6 & .938 & .468 \\
Pragmático & 6 & 1.704 & .120 & 6 & .345 & .913 & 6 & .345 & .913 & 6 & .632 & .705 \\
\hline
\end{tabular}

Nota: Grados de Libertad; EA: Estilo de Aprendizaje. ESO: Educación Secundaria Obligatoria. 
Como se observa en la tabla 12 existen algunas diferencias significativas entre las variables dependientes "Estilos de Aprendizaje" y las independientes "beneficios como tutorizado". En el Estilo activo con: la tutorización en estudio ( $\mathrm{p}=.008)$, la tutorización en acompañamiento $(\mathrm{p}=.008)$ y la tutorización en trabajos $(\mathrm{p}=.008)$. En el Estilo teórico con: la tutorización en estudio $(p<.001)$, la tutorización en acompañamiento $(p=.017)$ y la tutorización en trabajos ( $\mathrm{p}=.017$ ). Por lo que se rechaza parcialmente la Hipótesis 3.

En la tabla 13 recogemos el análisis de varianza de las variables dependientes "Estilos de Aprendizaje" y las variables independientes "beneficios como tutor".

Tabla 13

Análisis de varianza para la variable dependiente EA activo, reflexivo, teórico y pragmático, y las variables independientes relacionadas con los "beneficios como tutor" del alumnado de la ESO

\begin{tabular}{lccccccccc}
\hline \multirow{2}{*}{ EA } & \multicolumn{3}{c}{ Tutor de tareas } & \multicolumn{3}{c}{ Tutor de lectura } & \multicolumn{3}{c}{ Tutor de estudio } \\
\cline { 2 - 10 } & GL & F & p & GL & F & p & GL & F & p \\
\hline Activo & 6 & .926 & .476 & 6 & 2.354 & .031 & 6 & 1.490 & .181 \\
Reflexivo & 6 & 2.541 & .021 & 6 & .869 & .498 & 6 & 6.935 & .001 \\
Teórico & 6 & 1.356 & .232 & 6 & 1.126 & .347 & 6 & 2.170 & .046 \\
Pragmático & 6 & 1.098 & .363 & 6 & 1.031 & .405 & 6 & 2.499 & .023 \\
\hline
\end{tabular}

Nota: GL: Grados de Libertad; EA: Estilo de Aprendizaje; ESO: Educación Secundaria Obligatoria.

La tabla 13 muestra algunas diferencias significativas entre los Estilos de Aprendizaje como variables dependientes y las variables relacionas con el compañero tutor como variables independientes. En el Estilo activo con tutor de lectura ( $p=.031)$. En el Estilo reflexivo con tutor de tareas $(p=.021)$ y con tutor de estudio $(p<.001)$. En el Estilo teórico con tutor de estudio $(\mathrm{p}=.046)$. Y en el Estilo pragmático con el tutor de estudio $(\mathrm{p}=.023)$. Por lo que se rechaza parcialmente la Hipótesis 4.

En la tabla 14 se recoge el análisis de varianza de las variables dependientes "Estilos de Aprendizaje" y las variables independientes "problemas con la tutorización".

Tabla 14

Análisis de varianza para las variables dependientes EA activo, reflexivo, teórico y pragmático, y las variables independientes relacionadas con los "problemas con la tutorización" del alumnado de la ESO

\begin{tabular}{lllllll}
\hline EA & \multicolumn{3}{l}{ Problemas de lectura } & \multicolumn{3}{c}{ Problemas de comprensión } \\
\cline { 2 - 7 } & GL & F & $\mathbf{p}$ & GL & F & p \\
\hline Activo & 6 & 1.533 & .167 & 6 & 1.976 & .073 \\
Reflexivo & 6 & 1.815 & .096 & 6 & 1.134 & .341 \\
Teórico & 6 & 1.197 & .308 & 6 & .634 & .727 \\
Pragmático & 6 & .654 & .687 & 6 & 1.873 & .074 \\
\hline
\end{tabular}

Nota: GL: Grados de Libertad; EA: Estilo de Aprendizaje. ESO: Educación Secundaria Obligatoria. 
Como se advierte en la Tabla 14 no existen diferencias significativas entre la variable dependientes "Estilos de Aprendizaje" y las características del compañero tutor que hemos denominado como "problemas con la tutorización", por lo que se acepta la Hipótesis 5.

\section{Discusión}

Este trabajo ha tenido como objetivo principal relacionar los constructos "Estilo de Aprendizaje" y "compañero tutor", pues ambas líneas de investigación se vinculan con el desarrollo de los procesos de aprendizaje. Un análisis conjunto de ambos temas ayuda a potenciar las estrategias tendentes al desarrollo del aprendizaje en el alumnado. Además, esta investigación ha tenido dos objetivos secundarios, el primero, conocer el EA del grupo de alumnos de ESO en España, un ciclo formativo crítico en el desarrollo cognitivo del adolescente y, el segundo, avanzar en el estudio empírico de las características del compañero tutor.

Así, hemos podido comprobar que el EA característico del alumnado de la ESO es reflexivo. Este resultado coincide con estudios que analizaron los Estilos en este mismo nivel educativo (Quintanal y Gallego, 2011) y, también, con investigaciones en otros niveles educativos como, por ejemplo, en bachillerato (Adán, 2002; Santibáñez, Adán, Gil y Sáenz, 2004) y en la universidad (Alonso, 1992). Asimismo, el Estilo activo fue predominante en los estudiantes de matemáticas (Nevot, 2004). Estos resultados vienen a señalar que los alumnos responden a la exigencia del medio educativo en el que se encuentran. Pues, en la Enseñanza Secundaria fomenta la escucha y la observación, rasgos propios del Estilo reflexivo, frente a la espontaneidad que caracteriza al Estilo activo y la capacidad de experimentar y resolver problemas que caracteriza al Estilo pragmático.

En relación al estudio sobre la educación entre pares o compañero tutor se ha comprobado que los alumnos tienen una percepción positiva acerca de los beneficios de esta estrategia de aprendizaje, especialmente cuando se actúa como tutorizado ("Cuando mis compañeros o compañeras me ayudan a estudiar comprendo mejor lo que estoy estudiando") y en menor medida como tutor ("Cuando ayudo a un compañero o compañera a hacer sus deberes aprendo mejor"). Aunque también se observa que la tutorización puede generar problemas de aprendizaje ("Cuando leo una actividad con un compañero o compañera entiendo peor la actividad que estoy leyendo"). Esto vendría a señalar que para llevar a cabo esta estrategia de intervención a través de la figura del compañero tutor, parece necesario realizar análisis previos para controlar en qué medida esta estrategia de aprendizaje es propicia para el alumno y, asimismo, si el alumno es más favorable a ser tutor o tutorizado (Topping, 2000).

En el estudio sobre la varianza, que corresponden con las relaciones entre los Estilos de Aprendizaje y las variables relacionadas con las características del compañero tutor, no se han encontrado diferencias significativas en la mayoría de las situaciones. En la primera, que hemos denominado como "tareas de tutor", no se han encontrado en ninguna de las tareas y en ninguno de los Estilos, el apoyo que se puede llevar a cabo, no se relaciona con el Estilo de Aprendizaje. En la segunda, "beneficios como tutorizado", se han encontrado fundamentalmente en el Estilo activo. Estos resultados 
vendrían a señalar que los discentes con un EA activo podrían estar más predispuestos a este tipo de estrategia de aprendizaje. Como se ha señalado, este tipo de alumnado tiene una mayor implicación en nuevas experiencias y en general las personas son más abiertas al aprendizaje, lo que podría explicar las diferencias significativas. En la tercera, "beneficios como tutor", se encuentra una diferencia significativa en los casos de los EA reflexivo y pragmático, y la opción "tutor de estudio", entendemos que por las características del Estilo reflexivo, más centrado en el estudio y reflexión esta diferencia es razonable. En la cuarta y última, "problemas con la tutorización", no se han encontrado diferencias significativas, todos los Estilos pueden generar problemas.

Con todo lo señalado, se pueden destacar tres conclusiones clave de este estudio. Primera conclusión clave, al igual que en otras investigaciones (e.g., Quintanal y Gallego, 2011) se ha comprobado que el EA del alumnado de Educación Secundaria es reflexivo. De este resultado se obtienen dos importantes consecuencias. La primera, se refiere a que el alumno que no tenga un EA predominante reflexivo puede tener mayores problemas de fracaso escolar (Antelm y Gil, 2013), pues su EA no se ajusta a las exigencias del contexto educativo, más proclive a favorecer el Estilo reflexivo (Santibáñez et al., 2004). La segunda, se refiere a la necesidad de mejorar los Estilos Pragmático y Activo de este tipo de alumnado, con la finalidad de desarrollar de forma óptima su proceso de aprendizaje (Alonso, 1992).

Segunda conclusión clave, se ha probado que el alumnado de Educación Secundaria percibe el compañero tutor como una eficiente estrategia de aprendizaje. Sin embargo, se ha comprobado que en general los alumnos no se apoyan mayoritariamente en esta figura para mejorar su proceso de aprendizaje.

Tercera conclusión clave, se han comprobado diferencias significativas en la relación entre el EA y el compañero tutor, en algunos Estilos de Aprendizaje, especialmente en el Estilo activo. Por ello, se podría pensar que la figura del compañero tutor sería más apropiada para el desarrollo del EA activo.

Para terminar, en la actualidad, se presta especial atención a los modelos de enseñanza integradores en el aula (Buendía, Expósito, Aguadez y Sánchez, 2015; CecchiniEstrada, Méndez-Giménez y Fernández-Río, 2014) así como de inclusión en la escuela. La escuela inclusiva postula por encontrar estrategias que permitan atender a la diversidad educativa del alumnado (Pujolàs, 2003). El conocimiento del EA y la tutoría entre iguales, pueden ayudar al logro de un aprendizaje competencial y significativo, así como la integración del alumnado en el aula.

\section{Referencias}

Adán, I. (2002). Estilos de Aprendizaje, Modalidades de Bachillerato y rendimiento académico. Tesis doctoral inédita, UNED. Madrid: UNED.

Álvarez, V., Rodríguez, A, García, E., Gil, J., Romero, S., Padill, M.T., García, J., \& Correa, J. (2002). La atención a la diversidad en los centros de enseñanza secundaria: Estudio descriptivo de la provincia de Sevilla. Revista de Investigación Educativa, 20(1), 225-245. Recuperado de http://revistas.um.es/rie/article/viewFile/97631/93681 
Alonso C.M. (1992). Estilos de Aprendizaje: Análisis y diagnóstico en estudiantes universitarios. Tesis doctoral, Universidad Complutense de Madrid. Madrid: Universidad Complutense.

Alonso, C., Gallego, D., \& Honey, P. (1994). Los estilos de aprendizaje. Procedimientos de diagnóstico y mejora. Bilbao: Mensajero.

Antelm, A. M., \& Gil, A. J. (2013). El Estilo de Aprendizaje del alumnado en riesgo de abandono escolar. En A. Fidalago \& M.L. Sein-Echaluce (Eds.). Aprendizaje, Innovación y Creatividad (pp. 630-634). Madrid: Fundación General de la Universidad Politécnica de Madrid.

Armstrong, S.J., Cools, E., \& Sadler-Smith, E. (2012). Role of cognitive styles in business and management: Reviewing 40 years of research. International Journal of Management Reviews, 14, 238-262. doi: https://doi.org/10.1111/j.1468-2370.2011.00315.x

Buendía, L., Espósito, J., Aguadez, E.M., \& Sánchez, Ch. A. (2015). Análisis de la convivencia escolar en las aulas multiculturales de Educación Secundaria. Revista de Investigación Educativa, 33(2), 303-319. doi: https://doi.org/10.6018/rie.33.2.211491

Cecchini-Estrada, J.A., Méndez-Giménez, A., \& Fernández-Río, J. (2014). Análisis de un modelo integrador del aprendizaje: relaciones entre variables contextuales y meta creencias del alumnado de Secundaria. Aula Abierta, 42, 90-97. doi: https:// doi.org/10.1016/j.aula.2014.05.003

Cheng, Y.C., \& Ku, H.Y. (2009). An investigation of the effects of reciprocal peer tutoring. Computers in Human Behavior, 25, 40-49. doi: https://doi.org/10.1016/j.chb.2008.06.001

Cronbach, L. J. (1951). Coefficient alpha and the internal structure of tests. Psychometrika, 16, 297-334. Recuperado de https://link.springer.com/article/10.1007\%2FBF0231055 5? LI=true

De Backer, L., Van Keer, H., \& Valcke, M. (2012). Exploring the potential impact of reciprocal peer tutoring on higher education students' metacognitive know ledge and metacognitive regulation. Instructional Science, 40, 559-588. doi: https://doi. org/10.1007/s11251-011-9190-5

De Backer, L., Van Keer, H., \& Valcke, M. (2015). Exploring evolutions in reciprocal peer tutoring groups' socially shared metacognitive regulation and identifying its metacognitive correlates. Learning and Instruction, 38, 63-78. doi: https://doi. org/10.1016/j.learninstruc.2015.04.001

Dunn, R., Dunn, K., \& Price, G. (1975). The Learning Style Inventory. Lawrence, KS: Price Systems.

Dunn, R., Dunn, K., \& Price, G. (1989). Learning Styles Inventory (LSI): An Inventory for the Identification of How Individuals in Grades 3 through 12 Prefer to Learn. Lawrence, KS: Price Systems.

Durán, D. (coord.) (2006). Tutoría entre iguales, algunas prácticas. Monográfico de Aula de Innovación Educativa, 153-154, 7-39. doi: https://doi.org/10.13140/RG.2.1.4440.7440

Durán, D. \& Monereo, C., (2012). Entramados. Métodos de aprendizaje cooperativo y colaborativo. Barcelona: Horsori.

Durán, D., \& Vidal, V. (2004). Tutoría entre iguales: de la teoría a la práctica. Barcelona: Grao.

Fierro, A. (1993). Relaciones sociales en la adolescencia. En C. Coll, J. Palacios, \&A. Marchesi (Comps.). Desarrollo psicológico y educación, i. psicología de la educación (pp. 339-340). Madrid: Alianza. 
Fleming, S., Mckee, G., \& Huntley-Moore, S. (2011). Under graduate nursing students' learning styles: A longitudinal study. Nurse Education Today, 31, 444-449. doi: https:// doi.org/10.13140/RG.2.1.4440.7440

García, M.C. (2003). La medición de la estructura organizativa. Revista Europea de Dirección y Economía de la Empresa, 12(3), 163-176. Recuperado de https://dialnet. unirioja.es/servlet/articulo?codigo $=638879$

Gil, A.J., \& Sánchez, A. (2012). El Estilo de Aprendizaje del alumnado que cursa los Programas de Cualificación Profesional Inicial. En F. Guerra, R. García-Ruiz, N. González, P. Renés, \& A. Castro (Coords.). Estilos de Aprendizaje: Investigaciones y Experiencias. Santander: Universidad de Cantabria.

Gillies, R.M., \& Ashman, A.F. (2003). An historical review of the use of groups to promote socialization and learning. En R.M. Gillies, \& A. F. Ashman (Eds.). Cooperative learning: the social and intellectual outcomes of learning in groups (pp. 1-18). London: Routledgefalmer.

Ginsburg-Block, M., \& Fantuzzo, J. W. (1997). Reciprocal peer tutoring: an analysis of "teacher" and "student" interactions as a function of training and experience. School Psychology Quarterly, 12(2), 134-149. doi: http://dx.doi.org/10.1037/h0088955

Gregorc, A. (1982). Gregorc Style Delineator. Maynard, MA: Gabriel Systems.

Honey, P., \& Mumford, A. (1986).The Manual of Learning Styles. Maidenhead, Berskshire: Ardingly House.

Kolb, D. (1976). The Learning Style Inventory: Self-Scoring Test and Interpretation. Boston: McBer \& Company.

Kolb, D. (1984). Experiential learning: Experience at the Source of Learning and Development. New Jersey: Prentice Hall.

Li, M., \& Armstrong, S.J. (2015). The relationship between Kolb's experiential learning styles and Big Five personality traits in international managers. Personality and Individual Differences, 86, 422-426.

Loke, A.J.T.Y., \& Chow, F.L.W. (2007). Learning partnership - the experience of peer tutoring among nursing students: A qualitative study. International Journal of Nursing Studies, 44, 237-244. doi: https://doi.org/10.1016/j.ijnurstu.2005.11.028

Moya, M.V., Hernández, J.R., Hernández, J.A., \& Cózar, R. (2011). Análisis de los estilos de aprendizaje y las TIC en la formación personal del alumnado universitario a través del cuestionario REATIC. Revista de Investigación Educativa, 29(1), 137-156. Recuperado de https://digitum.um.es/xmlui/bitstream/10201/45267/1/Analisis\%20 de $\% 201$ os\%20estilos\%20de\%20aprendizaje\%20y\%20las\%20TIC\%20en \%20la\%20 formacion $\% 20$ personal $\% 20 \mathrm{del} \% 20$ alumnado $\% 20$ universitario $\% 20 \mathrm{a} \% 20$ traves $\% 2$ 0del\%20cuestionario\%20REATIC.pdf

Nevot, A. (2004). Enseñanza de las Matemáticas basada en los Estilos de Aprendizaje. Boletín de la Sociedad Española de Matemática Aplicada, 28, 169-184.

Nunnally, J. (1978). Psychometric theory. New York: McGraw-Hill.

Ormrod, J.E. (1999). Human learning (3rd Ed.). Upper Saddle River, NJ: Prentice Hall. Oxford, R. (1993): Style Analysis Survey (SAS). Tuscaloosa, AL: University of Alabama. Pérez, C. (2004).Técnicas de Análisis Multivariante de Datos. Madrid: Pearson.

Pujolàs, P. (2003). Aprendre junts alumnes diferents. Vic: Eumo. 
Quintanal, F., \& Gallego, D. J. (2011). Incidencia de los Estilos de Aprendizaje en el rendimiento académico de Física y Química de Secundaria. Revista de Estilos de Aprendizaje, 8(8), 198-223. Recuperado de http://www2.uned.es/ revistaestilosdeaprendizaje/numero_8/articulos/1sr_8_articulo_11.pdf

Riechmann, S., \& Grasha, A. (1974). A rational approach to developing and assessing the construct validity of the Student Learning Style Scales Instrument. Journal of Psychology, 87, 213-223. doi: https://doi.org/10.1080/00223980.1974.9915693

Rittschof, K. A., \& Griffin, B. W. (2001). Reciprocal peer tutoring: re-examining the value of a co-operative learning techniques to college students and instructors. Educational Psychology, 21(3), 313-331. Recuperado de http://ww.cfder.org/ uploads/3/0/4/9/3049955/reciprocal_peer_tutoring_re-examining_the_value_of_a_ cooperative_learning_technique_to_college_students_and_instructors.pdf

Rodríguez, M.C. \& Vázquez, E. (2013). Fortalecer estilos de aprendizaje para aprender a aprender. Revista Estilos de Aprendizaje, 11(11), 19-37. Recuperado de http://www2. uned.es/revistaestilosdeaprendizaje/numero_11/articulos/articulo_02.pdf

Santibáñez, J., Adán, I., Gil, A.J., \& Sáenz de Jubera, M. (2004). Estrategias didácticas y estilos de Aprendizaje, según sexo y modalidad de Bachillerato cursada. En C.M. Alonso y D.J. Gallego (Eds.): Actas I Congreso Internacional de Estilos de Aprendizaje. Madrid: Anaya.

Soflano, M., Connolli, T. M., \& Haney, T. (2015). Learning style analysis in adaptive GBL application to teach SQL. Computers $\mathcal{E}$ Education, 86, 105-119. doi: https://doi. org/10.1016/j.compedu.2015.02.009

Topping, K. (2000). Tutoring. Geneva, Switzerland, International Bureau of Education and the International Academy of Education. Serie Prácticas educativas, 5. [www. ibe.unesco.org].

Fecha de recepción: 24 de julio de 2015.

Fecha de revisión: 10 de mayo de 2017.

Fecha de aceptación: 10 de mayo de 2017. 
